\title{
Beam-Beam Effects for the PEP-II B Factory ${ }^{\dagger}$
}

\author{
Miguel A. Furmar \\ Accelerator \& Fusion Research Division \\ Lawrence Berkeley Laboratory, Berkeley, CA 94720, U.S.A. \\ and \\ Jennifer R. Eden \\ Department of Geophysics \\ University of Washington, Seattle, WA 98195, U.S.A.
}

\begin{abstract}
We present a summary of a fairly extensive study of beam-beam issues that arise in the design of PEP-II. Most of these studies are carried out with "strong-strong" multiparticle tracking simulations. We focus on: choice of nominal beambeam parameter, strength of the parasitic collisions, injection issues, performance and tradeoffs with unequal beam-beam parameters. We comment only briefly on beam lifetime. We conclude that: the beams are sufficiently well separated so that the parasitic collisions are effectively weak; that small inequalities in the beam-beam parameters may imply more comfortable performance margins; and that vertical injection with vertical beam separation is more comfortable than horizontal.
\end{abstract}

\section{INTRODUCTION}

The proposed PEP-II B Factory is an asymmetric $\mathrm{e}^{+}-\mathrm{e}^{-}$ collider with a design luminosity of $3 \times 10^{33} \mathrm{~cm}^{-2} \mathrm{~s}^{-1}$ whose primary purpose is the detailed study of the $B$ meson system. The two rings have the same circumference and intersect at only one interaction point (IP). In its present conception the low-energy ring (LER) contains the positrons, with an energy of $3.1 \mathrm{GeV}$, while the high-energy ring (HER) contains the electrons, with an energy of $9 \mathrm{GeV}$. Although the interaction region (IR) design allows for the possibility of crab crossing with a finite angle, in the current design the beams collide head-on and are magnetically separated in the horizontal plane. Full details of the design are contained in the Conceptual Design Keport [1] and in the Design Update Report [2]. The current PEP-II design is slightly different from that described in these reports: the beta functions of the HER at the IP are now $\beta_{x}{ }^{*} / \beta_{y}{ }^{*}=50 / 2 \mathrm{~cm}$ rather than $75 / 3 \mathrm{~cm}$, and the beam orbit separation at the first parasitic collision is now $d=3.5$ $\mathrm{mm}$ rather than $2.8 \mathrm{~mm}$.

The bulk of the beam-beam studies carried out to date have set a priority on demonstrating the feasibility of attaining or exceeding a short-time-average luminosity of $3 \times 10^{33} \mathrm{~cm}^{-2}$ $\mathrm{s}^{-1}$. The short-time-average luminosity is determined by the

\footnotetext{
†Work supported by the Director, Office of Energy Research, Office of High Energy and Nuclear Physics, High Energy Physics Division, U.S. Dept. of Energy, under Contract No. DE-AC0376 SF00098.
}

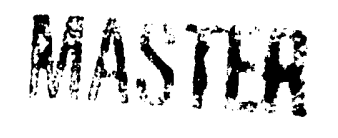

dynamics of the beam core, which is studied effectively with "strong-strong" or "weak-strong" simulations neglecting all lattice nonlinearities. This linear-lattice approximation is legitimate for these purposes on account of the good dynamicaperture properties of the PEP-II. Previous experiments and simulations [3] provide justification for this approximation once a good working point is adopted. On the other hand, the beam lifetime is determined by the long-time dynamics of the tails of the beam. This dynamics is far more complicated, and is generally expected to be sensitive to lattice nonlinearities. For this reason, and because it is impossible to have good average luminosity with poor peak luminosity, we have concentrated first on the dynamics of the beam core. Recent preliminary results [4], however, indicate acceptable lifetime.

In our simulations we typically represent the bunches by 256 "superparticles" that are initially Gaussian-distributed in 6-dimensional phase space, and we run for five damping times (we have carried out spot-checks with more superparticles). Thick-lens effects during the beam-beam collision are taken into account by dividing up the bunches into five slices. As time progresses, the distributions deviate a bit from Gaussian; however, for the purposes of computing the beam-beam kick, the time-dependent rms beam sizes are fed into the formula for the kick corresponding to a Gaussian distribution [5].

Due to the necessary approximations that are made, we cannot, in general, take our results to be quantitatively accurate. However, we believe that qualitative comparisons between results for different parameter values do provide valid guidance for desirable or undesirable changes in parameters. This is the philosophy that underlies the interpretation of our beam-beam studies, particularly multiparticle simulations.

\section{BEAM-BEAM PARAMETER}

The PEP-II design specifies a rather conservative value for the nominal beam-beam parameter, namely $\xi_{0}=0.03$ (all four beam-beam parameters are equal). The subscript 0 denotes "nominal," by which we mean "in the limit when the beambeam interaction is negligible." Simulation results in the absence of parasitic collisions (PCs) $[1,2]$ indicate that the dynamics behaves close to nominal (i.e., beam blowup is relatively small) up to $\xi_{0}=0.06-0.08$ provided a "good" working point is adopted (limited tune scans have suggested a working point with fractional tunes $(0.64,0.57)$ for both beams, which we have adopted for the present purposes; we have not tried to optimize the working point methodically). 


\section{PARASITIC COLLISIONS}

The large value required for the luminosity, coupled with other constraints, implies the need for many bunches, each of which has "normal" bunch current and emittance. This, in turn, implies short bunch spacing; thus, if the main collision is head-on, the bunches experience parasitic collisions in the IR. To be precise, each bunch experiences four PCs on either side of the IP (the optics and the geometry is symmetric about the IP in this region). The PCs on either side of, and closest to, the IP overwhelm the others, so we have only taken into account these first PCs in our simulations. The separation of the beam orbits at this point is $d=3.5 \mathrm{~mm}$, corresponding to a normalized separation $d / \sigma_{0 x_{1}+}=11.8$, where $\sigma_{0 x_{1}+}$ is the local nominal horizontal rms beam size of the LER (a normalization to the local HER beam size would yield the larger value of 14.4). In gerieral, $\mathrm{PC}$ induce tune shifts and tune spreads that can cause significant beam blowup. On the other hand, if the separation $d$ is large enough, all effects from the PCs disappear. Simulations $[1,2,6,7,8,9]$ in which the PC separation $d$ is taken as a free parameter show that the PC becomes essentially weak provided $d / \sigma_{0 x,+} \geq 7$. Thus the actual separation in the PEP-II design is comfortably large and the PCs are effectively weak. For the nominal value of the PC separation, the beam blowup has an inconsequential reduction of the luminosity to a dynamical value $\mathscr{L} \leqslant 2.9 \times 10^{33} \mathrm{~cm}^{-2}$ $\mathrm{s}^{-1}$.

When $d$ is varied as an independent parameter, the tunes sampled by the beam core also vary due to the $d$-dependence of the PC tune shift. This spurious effect can be compensated for by appropriate changes in the bare lattice tunes. Simulations show that, when this compensation is in effect, the blowup curves look smoother, although the qualitative shape is the same [10], and the conclusions drawn from the uncompensated simulations remain unaltered.

\section{VARIANTS}

\subsection{Larger $\xi_{0}$}

We have examined $[1,2,6,8,9]$ the consequences of increasing $\xi_{0}$ to 0.05 . We have done this in two ways: (a) by increasing the bunch current by a factor of $5 / 3$ at fixed nominal emittance, and (b) by decreasing the emittances by a factor of $3 / 5$ at fixed bunch current. In case (a) the simulations show that the onset of substantial beam blowup occurs for $d / \sigma_{0 x,+} \leqslant 9$ as $d$ decreases. The beam blowup factor at the nominal value of $d$ is larger than for 0.03; however, although the degradation of the luminosity relative to its nominal value, $\mathscr{L}_{0}=8.33 \times 10^{33} \mathrm{~cm}^{-2} \mathrm{~s}^{-1}$, is more significant, the resultant absolute dynamical value, $\mathscr{L} \leqslant 7 \times 10^{33} \mathrm{~cm}^{-2} \mathrm{~s}^{-1}$, is quite substantial. In case (b) the resultant nominal luminosity is $\mathscr{L}_{0}=5 \times 10^{33} \mathrm{~cm}^{-2} \mathrm{~s}^{-1}$, the beam sizes are a factor $\sqrt{3 / 5}$ smaller, and the normalized parasitic separation is thus $d / \sigma_{0 x,+}=\sqrt{5 / 3} \times 11.8=15.2$. The simulation shows that the beam blowup in this case reduces the luminosity to a dynamical value $\mathscr{L} \geq 4 \times 10^{33} \mathrm{~cm}^{-2} \mathrm{~s}^{-1}$. In this approach, the onset of substantial beam blowup also occurs for $d / \sigma_{0 x},+$ $\leqslant 9$ as $d$ decreases. We conclude that increasing $\xi_{0}$ by decreasing the emittance at fixed bunch current is safer, although less effective, than increasing the bunch current at fixed emittance.

\subsection{Larger bunch spacing}

One can increase the bunch spacing by $50 \%$ by filling every third RF bucket rather than every second bucket. In order to maintain $\xi_{0}$ and $\mathscr{L}_{0}$ at their original values, one must require that the bunch currents and nominal emittances of both beams also be increased by $50 \%$. The total beam current remains unchanged but the beam separation at the new parasitic crossing point is $\mathrm{d}=10.1 \mathrm{~mm}$ instead of $3.5 \mathrm{~mm}$, and $d / \sigma_{0 x,+}=20.1$ instead of 11.8 , which implies a much weaker parasitic collision. Simulations $[1,2,8,9]$ show that the beam blowup is slightly less for this case than for the previous case at the nominal value of $d / \sigma_{0 x,+}$. What is more imporiant is that the comfort margin for $d$ is significantly larger, since the value of 20.1 for $d / \sigma_{0 x},+$ is much larger than the "threshold" value of 7 .

If the bunches are injected every third bucket but the emittances and bunch currents have nominal values instead of being $50 \%$ larger [8], then the first PC is such that $d / \sigma_{0 x_{,+}}=$ 24.6 and therefore it is truly negligible. In this case the beamteam parameter at the IP is still $\xi_{0}=0.03$, but the luminosity is $\mathscr{L}_{0}=2 \times 10^{33} \mathrm{~cm}^{-2} \mathrm{~s}^{-1}$. This operating configuration might be used as a com.ortabli: intermediate stage in the commissioning of the machine, if necessary.

\subsection{Unequal bean-beam paraneters}

We have also explored consequences of breaking the equality of the beam-beam parameters [11]. To this end we have carried out simulations in two cases: in approach (a) we set $\xi_{0 x_{,}+}=\xi_{0 y_{,}+} \equiv \xi_{0+}$ and $\xi_{0 x,-}=\xi_{0 y_{1}-} \equiv \xi_{0-}$ with $\xi_{0+} \neq$

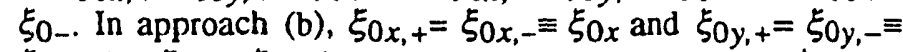
$\xi_{0 y}$ with $\xi_{0 x} \neq \xi_{0 y}$ (the subscripts + refer to the $\mathrm{e}^{+}$or $\mathrm{e}^{-}$ beams, respectively). In both cases we have maintained the pairwise equalit; of the rms beam sizes at the IP, and kept the nominal luminosity fixed at $3 \times 10^{33} \mathrm{~cm}^{-2} \mathrm{~s}^{-1}$. Other constraints were in effect. The simulations show that: (1) In both approaches only the vertical beam blowup is significant, and this blowup behaves smoothly as the beam-beam parameters move away from full equality. (2) In approach (a), the dynamics favors (i.e., beam blowup is lesser) $\xi_{0+} \approx 0.024$, $\xi_{0-} \approx 0.04$ over $\xi_{0+}=\xi_{0-}=0.03$. (3) In approach (b), the dynamics favors $\xi_{0 y} \approx 0.023, \xi_{0 x} \approx 0.04$ over $\xi_{0 x}=\xi_{0 y}=$ 0.03 . In both cases the dynamical value of the luminosity is slightly increased from the values corresponding to $\xi_{0 x}+=$ $\xi_{0 y,+}=\xi_{0 x,-}=\xi_{0 y,-}=0.03$. We conjecture that, if the beambeam parameters were chosen according to the preference expressed by the dynamics, the operation of the machine would perhaps be smoother. Of course, there are unfavorable implications for other areas of the design associated with these 
changes. For example, in both of these approaches, the total current in the LER is higher than in the nominal case.

\section{INJECTION SIMULATIONS}

The transient effects that follow the injection of the first $20 \%$ batch of the low-energy beam when the high-energy beam is fully stored have been studied by Chin [12]. If the beam is injected in the horizontal plane, the centroid oscillations can lead to almost head-on collisions at the PC locations, with substantial beam blowup, during the transient time. If injection is in the vertical plane, the effects from the PCs are weak. The conclusion is that vertical injection is favored over horizontal.

Assuming that a temporary orbit bump will be required for smooth injection, we have [13] looked at the effects on the dynamics of the fully stored beams after all injection transients have died down but before the orbit bump is tumed C.F. The optics of the PEP-II lattice is such that the kicking elements of a traditional orbit bump must be located at a distance $\geqq 2.5 \mathrm{~m}$ from the IP. This happens to be true for both rings whether the separation is vertical or horizontal. This means that all four parasitic collisions on either side of the IP would be encompassed by such an orbit bump. If the beams are separated horizontally, the simulation results indicate that the closed orbit bump must be tightly constrained by the lattice functions and phase advances of all the PC locations in order to maintain the beams well separated, while there is no such constraint in the vertical-separation case. In the unlikely event that an orbit bump encompassing only the IP and the first PC could be designed, a horizontal separation $3 \leq d_{x} / \sigma_{0 x}$ $\$ 10$ seems to be adequate for smooth injection. If the beams are vertically separated the dynamics is essentially determined by the main collision at the IP. In this case, a separation $d_{y}$ $\geq(1-2) \sigma_{0 x}$ is probably adequate for smooth injection. As a practical matter, the orbit separation must be - a few $\sigma_{0 x}$ 's in magnitude whether it is vertical or horizontal, for it to be effective; this conclusion from our simulations is consistent with available experience.

The horizontal-separation alternative does have the advantage that the simulations show no significant beam blowup when the beams are slowly brought into collision. In the vertical-separation case, on the other hand, the simulations show beam blowup of $\sim 75 \%$ in the vertical dimension when the beam centers come together by a distance $d_{y} \sim(1-2) \sigma_{0 y}$. Since PEP-II has conservative beam-stay-clear specifications, this temporary beam blowup seems a small price to pay, if any, for the added safety and simplicity of the vertical separation option.

\section{CONCLUSIONS}

(1) None of the simulation results show any indication that the energy asymmetry is a problem per se. Thus PEP-II (or any other asymmetric collider) does nut seem to be qualitatively different from a single-ring, symmetric machine from the perspective of beam-beam dynamics provided the beam-beam effects are properly balanced [14]. (2) A beam- beam parameter of 0.03 seems quite conservative for PEP-II. (3) The beams are sufficiently well separated that the PCs are effectively weak. (4) Small inequalities in the nominal beambeam parameters may imply more comfortable performance margins but not substantially better luminosity performance. (5) Vertical injection with vertical beam separation is more comfortable than horizontal.

\section{REFERENCES}

[1] "An Asymmetric B Factory Based on PEP: Conceptual Design Report," LBL PUB-5303/SLAC-372/CALT-681715/UCRL-ID-106426/UC-IIRPA-91-01, Feb. 1991.

[2] "PEP-II: An Asymmetric B Factory Design Update," Feb. 1992, unpublished.

[3] G. Jackson and R. Siemann, Nuclear Instruments and Methods A286, 17 (1990).

[4] T. Chen and J. Irwin, unpublished results.

[5] M. Bassetti and G. A. Erskine, CERN-ISR-TH/80-06.

[6] Y.-H. Chin, Proc. 1991 Part. Accel. Conf., San Francisco, May 1991, p. 213.

[7] J. L. Tennyson, ABC-29, April, 1991 (draft).

[8] J. R. Eden and M. A. Furntan, ABC-62/ESG Tech. Note 186, May, 1992.

[9] J. R. Eden and M. A. Furman, PEP-II/AP Note 292/ESG Tech-Note 210, October, 1992.

[10] J. R. Eden and M. A. Furman, PEP-II/AP Note 492/ESG Tech-Note 213, January, 1993.

[11] J. R. Eden and M. A. Furman, PEP-II/AP Note 6-92, ESG Tech Note-216, February, 1993.

[12] Y.-H. Chin, ABC-51/LBL-31434/ESG-158; LBL-32468; Proc. Intl. Conference on $B$ Factories: The State of the Art in Accelerators, Detectors, and Physics, Stanford, California, April 6-10, 1992, p.130.

[13] M. A. Furman, PEP-IU/AP Note 14-93/CBP Tech Note011, April 1993.

[14] Y.-H. Chin, in Beam Dynamics Issues of High Luminosity Asymmetric Collider Rings, Ed. Andrew M. Sessler, AIP Cor.ference Proceedings 214, p. 424 (1990); A. Garren et aì, Proc. 1989 Particle Accelerator Conf., Chicago, March 20 - 23, 1989, p. 1847. 

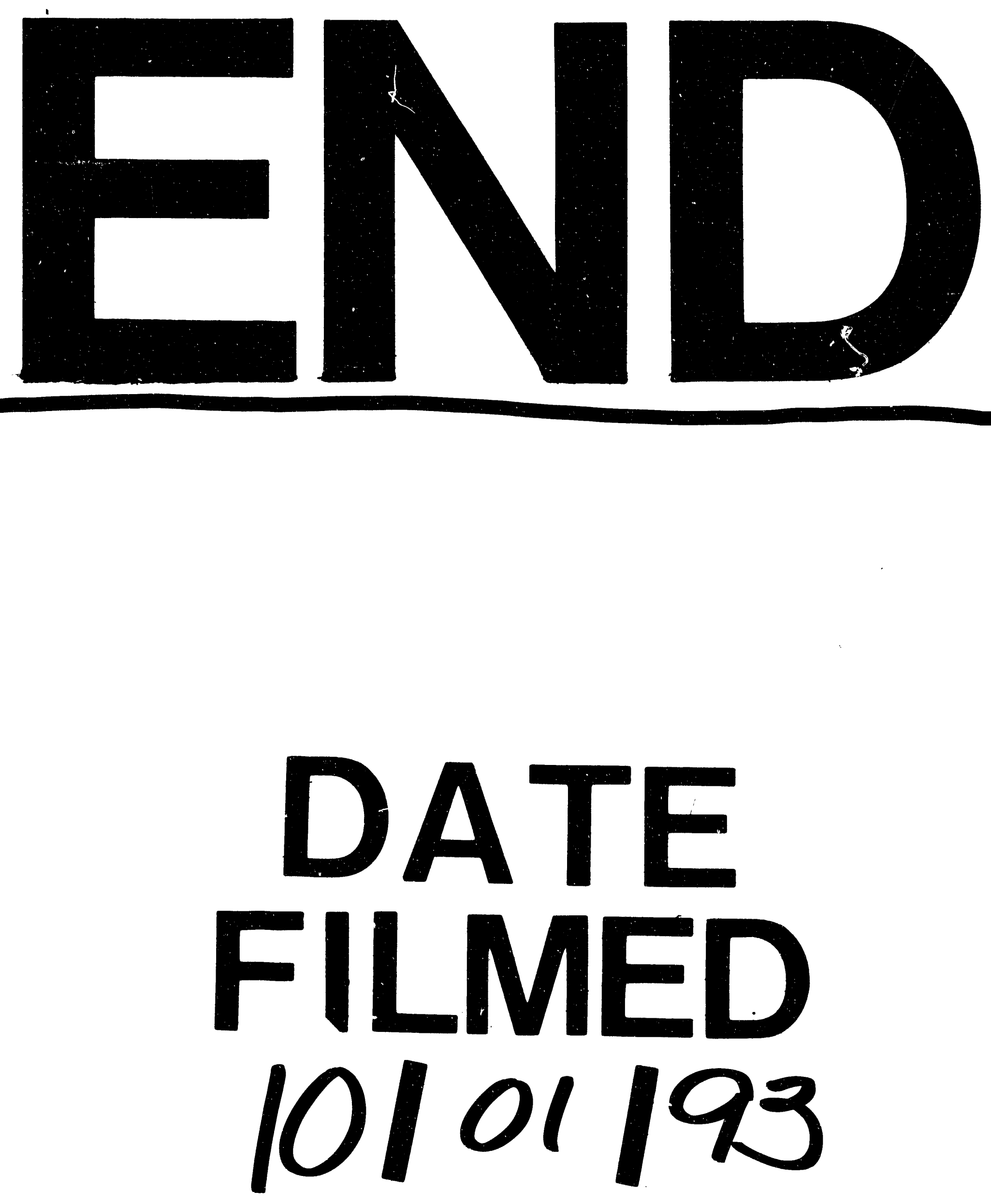


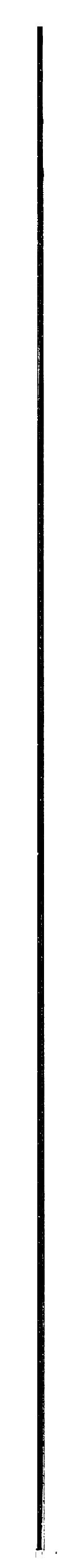

